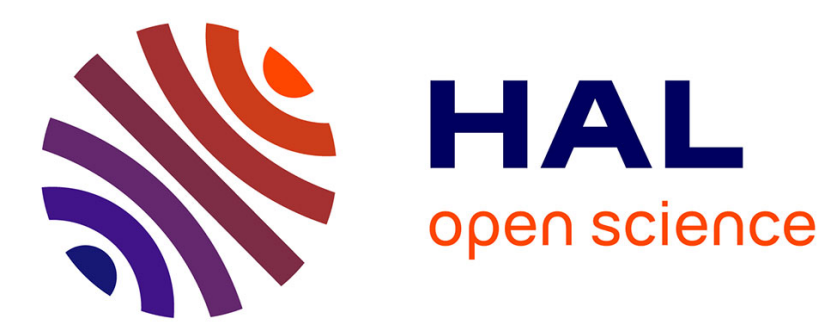

\title{
Lissajous-toric knots
}

\author{
Marc Soret, Marina Ville
}

\section{To cite this version:}

Marc Soret, Marina Ville. Lissajous-toric knots. Journal of Knot Theory and Its Ramifications, 2020, 29 (01), pp.2050003. 10.1142/S0218216520500030 . hal-03064345

\section{HAL Id: hal-03064345 \\ https://hal.science/hal-03064345}

Submitted on 15 Dec 2020

HAL is a multi-disciplinary open access archive for the deposit and dissemination of scientific research documents, whether they are published or not. The documents may come from teaching and research institutions in France or abroad, or from public or private research centers.
L'archive ouverte pluridisciplinaire HAL, est destinée au dépôt et à la diffusion de documents scientifiques de niveau recherche, publiés ou non, émanant des établissements d'enseignement et de recherche français ou étrangers, des laboratoires publics ou privés. 


\title{
Lissajous-toric knots
}

\author{
Marc Soret and Marina Ville
}

\begin{abstract}
A point in the $(N, q)$-torus knot in $\mathbb{R}^{3}$ goes $q$ times along a vertical circle while this circle rotates $N$ times around the vertical axis. In the Lissajous-toric knot $K(N, q, p)$, the point goes along a vertical Lissajous curve (parametrized by $t \mapsto(\sin (q t+\phi), \cos (p t+\psi)))$ while this curve rotates $N$ times around the vertical axis. Such a knot has a natural braid representation $B_{N, q, p}$ which we investigate here. If $\operatorname{gcd}(q, p)=1, K(N, q, p)$ is ribbon; if $\operatorname{gcd}(q, p)=d>1, B_{N, q, p}$ is the $d$-th power of a braid which closes in a ribbon knot. We give an upper bound for the 4-genus of $K(N, q, p)$ in the spirit of the genus of torus knots; we also give examples of $K(N, q, p)$ 's which are trivial knots.
\end{abstract}

\section{Introduction}

We study a class of knots generalizing torus knots, which we call Lissajoustoric: a torus knot is generated by a a circle rotating around an axis and a Lissajous-toric knot is generated by a Lissajous curve rotating around the axis. There are several ways of describing them.

\subsection{Lissajous-toric knots: various points of view}

\subsubsection{A description in $\mathbb{R}^{3}$}

We recall the description of the $(N, q)$-torus knot in $\mathbb{R}^{3}$ endowed with an orthonormal frame $O x y z$ (see for example $[\mathrm{Cr}] 1.5$ ). If $\Gamma$ is the circle of radius 1 centered at $(0,2,0)$ in the $y z$ plane, a point travelling along the knot goes $q$ times around $\Gamma$ while $\Gamma$ is rotated $N$ times around the axis $O z$. In the case of the Lissajous-toric knots, we replace the vertical circle by a vertical Lissajous curve: we take three integers $N, q, p$ with $(N, q)=(N, p)=$ 
1 and a real number $\phi$, and we define a $\operatorname{knot} K(N, q, p, \phi)$ as follows. Consider the curve $C_{q, p, \phi}$ given in a vertical plane by

$$
\begin{gathered}
t \in[0,2 \pi] \longrightarrow \mathbb{R}^{3} \\
t \mapsto(2+\sin (q t), 0, \cos (p(t+\phi)))
\end{gathered}
$$

and rotate $C_{q, p, \phi} N$ times around the axis generated by $(0,0,1)$. In Cartesian coordinates, we write the knot as

$$
(*)\left\{\begin{array}{l}
x=(2+\sin (q t)) \cos (N t) \\
y=(2+\sin (q t)) \sin (N t) \\
z=\cos (p(t+\phi))
\end{array}\right.
$$

\subsubsection{A description in the 3-dimensional cylinder}

We write $(*)$ above in cylindrical coordinates:

$$
\left\{\begin{array}{l}
\theta=N t \\
\rho=2+\sin (q t) \\
z=\cos (p(t+\phi))
\end{array}\right.
$$

Thus $K(N, q, p, \phi)$ is a closed $N$-braid which we can write in the 3 -cylinder $\mathbb{S}^{1} \times \mathbb{R}^{2}$ as follows :

$$
e^{i t} \mapsto\left(e^{N i t}, \sin (q t), \cos (p(t+\phi))\right)
$$

Note the similarity with the $(N, q)$-torus knot which is written in the 3 -sphere or the 3-cylinder as

$$
e^{i t} \mapsto\left(\frac{1}{\sqrt{2}} e^{N i t}, \frac{1}{\sqrt{2}} e^{q i t}\right)
$$

\subsubsection{Billiard curve in a solid torus}

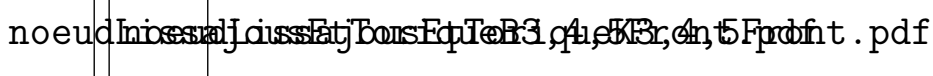

Figure 1: Perspective front view of $\operatorname{knots} B(3,4,5)$ and $K(3,4,5)$ 
Just as Billiard curves are equivalent to Lissajous knots (cf. [JP]) Lissajous toric knots are equivalent to billiard curves in a square solid torus, namely a cube where the top and bottom have been identified; C. Lamm introduced them in [La 1], see also the related [L-O]. Such billiard curves are parametrized similarly to Lissajous toric knots; the trigonometric functions are replaced by saw-tooth functions of the type $g(t):=2\left|t-[t]-\frac{1}{2}\right|$. and $h(t):=t-[t]$.

$$
C(N, p, q, \phi):\left(\begin{array}{cc}
{[0,2 \pi]} & \longrightarrow[-1,1]^{3} \\
t & \mapsto(g(N t), g(p(t+\phi)), h(q t))
\end{array}\right)
$$

C. Lamm noticed that these billiard curves in a solid torus do not depend on the phase up to mirror transformation and stated that, if $p$ and $q$ are mutually prime, the knot $K(N, q, p)$ is ribbon.

\subsubsection{Singularity knots of minimal surfaces}

We first encountered the $K(N, q, p, \phi)$ 's in $[\mathrm{S}-\mathrm{V}]$ when we studied the singularities of minimal disks in $\mathbb{R}^{4}$; having noticed that their knot types do not depend on the phase $\phi$ up to mirror transformation, we dropped the $\phi$ in the notation.

We consider a minimal, i.e. conformal harmonic, map $F: \mathbb{D} \longrightarrow \mathbb{R}^{4}$ where $\mathbb{D}$ is the unit disk in $\mathbb{C}$, with $d F(0)=0$, i.e. $F$ has a branch point at 0 . If moreover $F$ is a topological embedding, we can copy Milnor's construction of algebraic knots $([\mathrm{Mi}])$ and take the intersection of $F(\mathbb{D})$ with a small sphere centered at $F(0)$ : we obtain a minimal knot. Complex curves are a special case of minimal surfaces and the germ $z \mapsto\left(z^{N}, z^{q}\right)$ yields the $(N, q)$-torus knot. In $[\mathrm{S}-\mathrm{V}]$ the knots $K(N, q, p, \phi)$ 's came from germs of singularities of the type

$$
z \mapsto\left(\operatorname{Re}\left(z^{N}\right), \operatorname{Im}\left(z^{N}\right), \operatorname{Im}\left(z^{q}\right), \operatorname{Re}\left(e^{p i \phi} z^{p}\right)\right)
$$

with

$$
N<p, q
$$

In $[\mathrm{S}-\mathrm{V}]$ we called the $K(N, q, p)$ 's simple minimal knots; in the present paper we drop the assumption (5) and study these knots per se; Lissajous-toric is a more appropriate name for the general case. 


\subsection{Contents of the paper}

In $[\mathrm{S}-\mathrm{V}]$ we defined a braid $B_{N, q, p}$ naturally associated to the knot $K(N, q, p)$; we describe it here in much greater detail. We view $B_{N, q, p}$ as a collection of graphs of $N$ functions from $[\eta, 1+\eta]$ to $\mathbb{R}^{2}$; the purpose of the small positive number $\eta$ is to avoid crossing points at the endpoints of the interval.

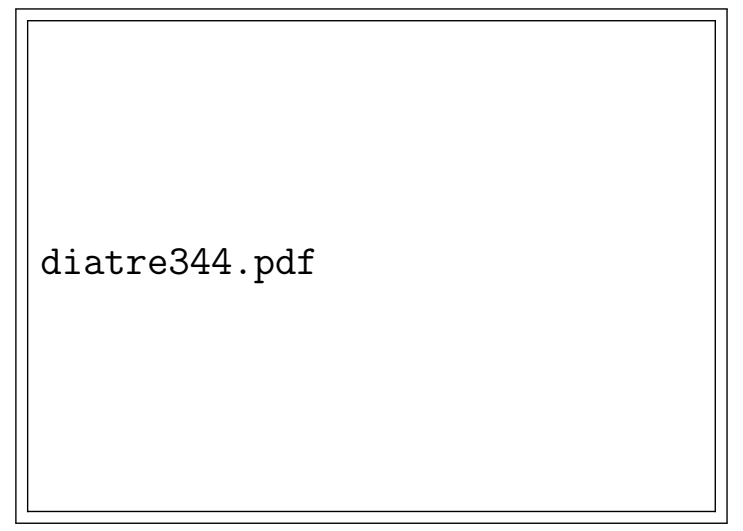

Figure 2: Braid shadow of $B(5, q, p)$

We prove in $\S 4.1$ below

Proposition 1. Let $d=\operatorname{gcd}(p, q), \tilde{q}=\frac{q}{d}, \tilde{p}=\frac{p}{d} ;$ then

$$
B_{N, q, p}=B_{N, \tilde{q}, \tilde{p}}^{d}
$$

Since $\tilde{q}$ and $\tilde{p}$ are mutually prime and since the knot type does not change if we interchange $p$ and $q$, we make the

Assumption 1. The numbers $p$ and $q$ are mutually prime and $q$ is odd.

In $\S 2$, we construct two braids $\alpha_{N, q, p}$ and $\beta_{N, q, p}$ of the form

$$
\alpha_{N, q, p}=\prod_{2 \leq 2 k \leq N-1} \sigma_{2 k}^{ \pm} \quad \beta_{N, q, p}=\prod_{1 \leq 2 k+1 \leq N-1} \sigma_{2 k+1}^{ \pm}
$$

where the $\sigma_{i}$ 's are the standard generators of the braid group $\mathbf{B}_{N}$ and the exponents \pm 1 of the $\sigma_{i}$ 's appearing in $\alpha$ and $\beta$ are given by simple formulae in $N, q, p$. 
We will state below the Main Theorem which expresses the braid $B_{N, q, p}$ as a product of the braids $\alpha_{N, q, p}, \alpha_{N, q, p}^{-1}, \beta_{N, q, p}$ and $\beta_{N, q, p}^{-1}$ as follows :

$$
B_{N, q, p}=Q_{N, q, p} \alpha_{N, q, p} Q_{N, q, p}^{-1} \beta_{N, q, p}
$$

where the $N$-braid $Q_{N, q, p}$ is also a product of $\alpha_{N, q, p}^{ \pm 1}$ 's and $\beta_{N, q, p}^{ \pm 1}$ 's. We illustrate the Main Theorem in $\S 3$ by going through the examples we gave in $[\mathrm{S}-\mathrm{V}]$ and we prove it in $\S 4$.

In the rest of the paper, we drop the Assumption 1 and study the topology of the knot. In $\S 5.1$, we reprove a theorem of Lamm:

Theorem 1. If $p$ and $q$ are mutually prime, the knot $K(N, q, p)$ is ribbon.

Corollary 1. If $d=\operatorname{gcd}(p, q)>1$, the knot $K(N, q, p)$ is periodic and its braid is the d-th power of a braid which closes in a ribbon knot.

Theorem 2. If $d=\operatorname{gcd}(p, q)$, the four-genus of $K(N, q, p)$ verifies

$$
g_{4}(K(N, q, p)) \leq \frac{(N-1)(d-1)}{2} .
$$

Remark 1. The right-hand side of (9) is the genus of the $K(N, d)$-torus knot (cf. $[K-M])$.

Remark 2. The inequality (9) can be strict: for example the knot $K(3,5,10)$ is $10_{123}$ which is slice.

There is one case where we know that (9) is an equality:

Proposition 2. Let $N, q, p$ be positive integers with $(N, q)=(N, p)=1$, $d=g c g(q, p)$ and let

$$
\begin{gathered}
\tilde{p}=\frac{p}{d} \quad \tilde{q}=\frac{q}{d} \\
\text { If } \quad \tilde{p}+\tilde{q} \equiv 0(2 N) \quad \text { or } \quad \tilde{p}-\tilde{q} \equiv 0(2 N)
\end{gathered}
$$

the knot $K(N, q, p)$ is represented by a quasipositive braid and its 4-genus is

$$
g_{4}(K(N, q, p))=\frac{(N-1)(d-1)}{2}
$$


Finally, replacing $t$ by $t+\pi$ in the expression of $K(N, q, p)$ given in $\S 1.1 .1$ yields

Proposition 3. If $p$ and $q$ have different parities (and thus $N$ is odd), then $K(N, q, p)$ is preserved by the involution

$$
(x, y, z) \mapsto(-x,-y,-z) .
$$

Hence it is positive strongly amphicheiral.

Some of the $K(N, q, p)$ 's are actually trivial knots; in $\S 6$ show:

Proposition 4. If $N$ and $q$ are mutually prime, the knots $K(N, q, q+N)$, $K(N, q, 1), K(N, q, 2 N q+1)$ and $K(N, q, 2 N q-1)$ are trivial.

Can we get all the trivial $K(N, q, p)$ 's this way? We did computer simulations using the braid software from the Liverpool knot group ([br]) and KnotPlot ([KP]): they told us that in some cases (the $K(4,5,$.$) 's for exam-$ ple) the answer is yes but in most cases the answer is no (see the lists of Jones polynomials at the end of the paper).

\section{Acknowledgment}

We are grateful to Moshe Cohen whose stimulating conversation prompted us to embark on this work.

\section{The structure of a simple minimal braid}

\subsection{Overview}

Here is an informal description of the contents of the Main Theorem.

There are $2 q$ values of $t$ in $[\eta, 1+\eta]$ (we call them crossing values) above which two or more of the $N$ graphs forming $B_{N, q, p}$ meet (at crossing points) and the data of all these crossing points make up the braid (see Figure 2); above each of the crossing values t's, the generators of the braid group $\mathbf{B}_{N}$ describing the corresponding crossing points are all even (i.e. of the form $\sigma_{2 k}^{ \pm 1}$ ) or all odd (i.e. of the form $\sigma_{2 k+1}^{ \pm 1}$ ). 
The set of crossing points of $B_{N, q, p}$ above a crossing value $t$ can be represented by one of the braids: $\alpha_{N, q, p}, \alpha_{N, q, p}^{-1}, \beta_{N, q, p}$ or $\beta_{N, q, p}^{-1}$ which were introduced in formula (7); thus $B_{N, q, p}$ is a product of the $\alpha_{N, q, p}$ 's and $\beta_{N, q, p}$ 's and of their inverses.

We order the $2 q$ crossing values $t_{1}<t_{2}<\ldots<t_{q}<\ldots<t_{2 q}$. Going from $t_{k}$ to $t_{k+1}$ changes $\alpha_{N, q, p}^{ \pm 1}$ into $\beta_{N, q, p}^{ \pm 1}$ or vice-versa. A formula gives us the exponent +1 or -1 of the $\alpha_{N, q, p}$ or $\beta_{N, q, p}$ above a given crossing point $t_{k}$ in terms only of $N, q, p$ and $k$.

Finally we notice that, if we have an $\alpha_{N, q, p}\left(\operatorname{resp} . \alpha_{N, q, p}^{-1}, \beta_{N, q, p}, \beta_{N, q, p}^{-1}\right)$ for $t_{k}($ with $k \neq q)$, we have a $\alpha_{N, q, p}^{-1}\left(\operatorname{resp} . \alpha_{N, q, p}, \beta_{N, q, p}^{-1}, \beta_{N, q, p}\right)$ for $t_{2 q-k}$ : this explains the presence of $Q_{N, q, p}$ and $Q_{N, q, p}^{-1}$ in the product (8).

\subsection{Statement of the structure theorem}

Main Theorem. Let $N, p, q$ be three integers such that $q$ is odd and $(p, q)=$ $(N, q)=(N, p)=1$; and let $A, B$ two integers such that

$$
2 N A+B q=1
$$

For $i \in\{1, \ldots, N-1\}$, we let

$$
\epsilon_{N, q, p}(i)=(-1)^{\left[\frac{p B i}{N}\right]}
$$

where [] denotes the integral part and we define

$$
\alpha_{N, q, p}=\prod_{2 \leq 2 i \leq N-1} \sigma_{2 i}^{\epsilon_{N, q, p}(2 i)} \quad \beta_{N, q, p}=\prod_{1 \leq 2 i+1 \leq N-1} \sigma_{2 i+1}^{\epsilon_{N, q}(2 i+1)}
$$

For $k \in\{1, \ldots, 2 q\}, k \neq q, k \neq 2 q$, we let

$$
\lambda_{N, q, p}(k)=(-1)^{\left[\frac{2 A p k}{q}\right]}
$$

Up to mirror transformation, the knot $K(N, q, p)$ is represented by the braid

$$
B_{N, q, p}=\underbrace{\alpha_{N, q, p}^{\lambda(1)} \beta_{N, q, p}^{\lambda(2)} \ldots \alpha_{N, q, p}^{\lambda(q-2)} \beta_{N, q, p}^{\lambda(q-1)}}_{Q_{N, q, p}} \alpha_{N, q, p} \underbrace{\beta_{N, q, p}^{-\lambda(q-1)} \alpha_{N, q, p}^{-\lambda(q-2)} \ldots \beta_{N, q, p}^{-\lambda(2)} \alpha_{N, q, p}^{-\lambda(1)}}_{Q_{N, q, p}^{-1}} \beta_{N, q, p}
$$

The $k$-th factor in this expression corresponds to the $k$-th crossing value $t_{k}$. 
Notice that the arithmetic formulae (14) and (16) can be written in terms of the Conway sign:

Definition 1. ([Co]) If $m$ and $n$ are two integers, $n$ is said to be positive (resp. negative) modulo $m$ if $n$ is congruent to an integer inside $\left(0, \frac{m}{2}\right)$ (resp. $\left.\left(0,-\frac{m}{2}\right)\right)$.

\section{Illustrations and examples}

In this section we go through the examples featured in [S-V] and we write their braid using the terminology of the Main Theorem.

We define three permutations of the crossing values $t_{k}, k \in\{1, \ldots, 2 q\}$ and their corresponding action on the blocks $\alpha$ and $\beta$ :

$$
\begin{aligned}
& T(k)=k+q, S(k)=2 q-k, R(k)=q-k \\
& T: \alpha \mapsto \beta, \beta \mapsto \alpha \\
& S: \alpha \mapsto \alpha^{-1}, \beta \mapsto \beta^{-1} \\
& R: \alpha \mapsto \beta^{-1}, \beta \mapsto \alpha^{-1}
\end{aligned}
$$

Since it is clear in each case of the following list what the $N, q, p$ are, we dropped the indices $N, q, p$.

- $N=3, q=4, p=5$ : square knot $3_{1} \# \overline{3}_{1}$

$$
Q \sigma_{2}^{-1} Q^{-1} \sigma_{1} \quad \text { where } \quad Q=\sigma_{2} \sigma_{1}^{-1} \sigma_{2}^{-1} \sigma_{1}
$$

- $N=3, q=4, p=7$ : trivial knot

$$
Q \sigma_{2} Q^{-1} \sigma_{1}^{-1} \quad \text { where } \quad Q=\left(\sigma_{2}^{-1} \sigma_{1}^{-1} \sigma_{2}^{-1}\right)^{2}
$$

- $N=3, q=4, p=10$ : figure eight knot

$$
B_{3,4,10}=B_{3,2,5}^{2}=\left(Q \sigma_{2} Q^{-1} \sigma_{1}^{-1}\right)^{2} \quad \text { where } \quad Q=\sigma_{2} \sigma_{1} \sigma_{2} \sigma_{1}
$$

- $N=3, q=5, p=7: 10_{155}$

$$
Q \sigma_{2}^{-1} Q^{-1} \sigma_{1}^{-1} \quad \text { where } \quad Q=\sigma_{2}^{-1} \sigma_{1} \sigma_{2}^{-1} \sigma_{1}
$$

Note that this knot verifies the assumptions of Theorem 2. 
- $N=3, q=5, p=10: 10_{123}$

$$
B_{3,5,10}=B_{3,1,2}^{5}=\left(\sigma_{2}^{-1} \sigma_{1}\right)^{5}
$$

- $N=3, q=7, p=8: 5_{1} \# \overline{5}_{1}$

$$
Q \sigma_{2}^{-1} Q^{-1} \sigma_{1} \quad \text { where } \quad Q=\sigma_{2} \sigma_{1}^{-1} \sigma_{2} \sigma_{1} \sigma_{2}^{-1} \sigma_{1}
$$

- $N=3, q=7, p=19: 14 N 11995$

$$
Q \sigma_{2} Q^{-1} \sigma_{1} \quad \text { where } \quad Q=\sigma_{2} \sigma_{1}^{-1} \sigma_{2}^{-1} \sigma_{1} \sigma_{2} \sigma_{1}^{-1}
$$

- $N=4, q=5, p=7: 5_{2} \# \overline{5}_{2}$

$$
Q \alpha Q^{-1} \beta \quad \text { where } \quad \alpha=\sigma_{2}^{-1} \quad \beta=\sigma_{1} \sigma_{3} \quad Q=\alpha^{-1} \beta^{-1} \alpha \beta
$$

- $N=4, q=5, p=13: 9_{46}$

$$
Q \alpha Q^{-1} \beta \quad \text { where } \quad \alpha=\sigma_{2} \quad \beta=\sigma_{1} \sigma_{3} \quad Q=\alpha \beta \alpha^{-1} \beta^{-1}
$$

- $N=5, q=6, p=22: 7_{7}$

$$
B_{5,6,22}=B_{5,3,11}^{2}=\left(Q \alpha Q^{-1} \beta\right)^{2} \quad \text { where } \alpha=\sigma_{2} \sigma_{4}^{-1} \quad \beta=\sigma_{1}^{-1} \sigma_{3} \quad Q=\alpha^{-1} \beta
$$

Figure 3: $B_{4,5,13}, A=4, \alpha \stackrel{\stackrel{\substack{b \\=}}{=}}{=}, \beta=\sigma_{1} \sigma_{3}, \lambda(k)=(-1)^{\left[\frac{2 k}{5}\right]}$

\section{TRE5622marina.pdf}

Figure 4: $B_{5,6,22}=B_{5,3,11}^{2}, \alpha=\sigma_{2} \sigma_{4}^{-1}, \beta=\sigma_{1}^{-1} \sigma_{3}$ 


\section{Proof of the Main Theorem on the simple minimal braid $B_{N, q, p}$}

K375.png

Figure 5: Graph of $\left\{\psi_{k}\right\}_{, k=1,2,3}$ of knot $K(3,7,5)$

We recall some facts from $[\mathrm{S}-\mathrm{V}]$. We endow $\mathbb{R}^{3}$ with coordinates $(t, y, z)$ : the braid is the collections of the graphs in $\mathbb{R}^{3}$ of the functions $\psi_{k}$ for $k=$ $1, \ldots, N$ :

$$
\begin{gathered}
\psi_{k}=\left(\psi_{k}^{(1)}, \psi_{k}^{(2)}\right):[\eta, 1+\eta] \longrightarrow \mathbb{R}^{2} \\
t \mapsto(y, z)=\left(\psi_{k}^{(1)}(t), \psi_{k}^{(2)}(t)\right)=\left(\sin \frac{2 \pi q}{N}(t+k), \cos \frac{2 \pi p}{N}(t+k+\phi)\right)
\end{gathered}
$$

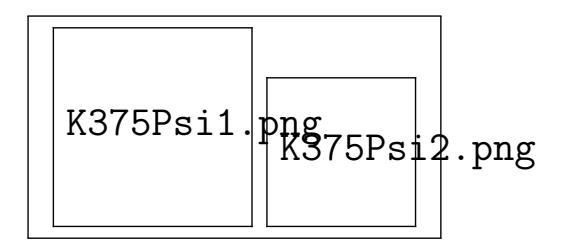

Figure 6: Graph of $\left\{\psi_{k}^{(1)}\right\}_{k=1,2,3}$ and $\left\{\psi_{k}^{(2)}\right\}_{k=1,2,3}$ of knot $K(3,7,5)$

\subsection{Periodic braids: proof of Proposition 1}

We now prove Proposition 1 (stated in 1.2). We divide the interval $[\eta, 1+\eta]$ into $d$ intervals

$$
I_{n}=\left[\frac{n}{d}+\eta, \frac{n+1}{d}+\eta\right], \quad n=0, \ldots, d-1 .
$$

After a change of variables $t \mapsto s=d t$, we see that the braid above an interval $I_{n}$ consists in the collection of graphs of the functions $[d \eta, 1+d \eta] \longrightarrow \mathbb{R}^{2}$

$$
s \mapsto\left(\sin \frac{2 \pi \tilde{q}}{N}(s+d k), \cos \frac{2 \pi \tilde{p}}{N}(s+d k+d \phi)\right)
$$


Since $(N, d)=1$, the map $k \mapsto k d(\bmod N)$ induces a permutation of $\{1, \ldots, N-1\}$; hence the piece of $B_{N, q, p}$ above $I_{n}$ is the collection of graphs above $[\eta, 1+\eta]$ of the functions

$$
s \mapsto\left(\sin \frac{2 \pi \tilde{q}}{N}(s+k), \cos \frac{2 \pi \tilde{p}}{N}(s+k+d \phi)\right)
$$

i.e. it is the braid $B_{N, \tilde{q}, \tilde{p}}$, representing the $\operatorname{knot} K(N, \tilde{q}, \tilde{p}, d \phi)$; this proves Proposition 1.

\subsection{Crossing values and crossing points of the braid}

The braid shadow is the projection of the braid onto the first two components $(t, y)$ of $\mathbb{R}^{2}$ i.e. the collection of the graphs of the $\psi_{k}^{(1)}$ 's.

A crossing point $P$ of the braid is the data of two different integers, $k, l$ with $0 \leq k, l \leq N-1$ and a number $t \in[\eta, 1+\eta]$ called a crossing value such that

$$
\psi_{k}^{(1)}(t)=\psi_{l}^{(1)}(t) \quad \text { i.e. } \quad \sin \left(\frac{2 \pi}{N} q(t+k)\right)=\sin \left(\frac{2 \pi}{N} q(t+l)\right) .
$$

There is a total of $(N-1) q$ crossing points, as in the case of the $(N, q)$ torus knot (where $q=p$ ).

A straightforward computation (cf. $[\mathrm{S}-\mathrm{V}]$ ) shows that, for a crossing point $P$ between the $k$-th and $l$-th strands of $B_{N, q, p}$, the corresponding crossing value $t$ verifies for some integer $m$

$$
t=-\frac{k+l}{2}+\frac{N}{4 q}(2 m+1)
$$

The sign $\Sigma(P)$ of a crossing point $P$ is signe.png

Figure 7: crossing numbers +1 and -1

$$
\Sigma(P)=\operatorname{sign} \text { of } \quad\left(\psi_{k}^{(2)}(t)-\psi_{l}^{(2)}(t)\right)\left(\psi_{l}^{(1) \prime}(t)-\psi_{k}^{(1) \prime}(t)\right)
$$


In $[\mathrm{S}-\mathrm{V}]$, we computed this $\Sigma(P)$ as:

$$
\Sigma(P)=(-1)^{m}(-1)^{\left[p \frac{m}{q}+\frac{p}{2 q}+\frac{2 p \phi}{N}\right]}(-1)^{\left[p \frac{k-l}{N}\right]}(-1)^{\left[q \frac{k-l}{N}\right]}
$$

where [ ] denotes the integral part.

\subsubsection{Determination of the crossing points above a given crossing value}

Let $t$ be a crossing value of $B_{N, q, p}$. We look for the $y$ 's such that $(t, y)$ is a crossing point of the braid shadow.

We derive from (21) the existence of at least one ordered pair of integers $(m, \mathfrak{s})$ such that

$$
t=-\frac{\mathfrak{s}}{2}+\frac{N}{4 q}(2 m+1) .
$$

(with $\mathfrak{s}=k+l)$. There can be several $(m, \mathfrak{s})$ 's verifying $(24)$ for the same crossing value $t$; however

$$
1 \leq \mathfrak{s}=k+l \leq 2 N-3
$$

which implies that there are at most two possible $(m, \mathfrak{s})$ 's (Lemma 1 below). We will see later that for $t$ and $(m, \mathfrak{s})$ given, a crossing point $(t, y)$ of the braid shadow above $t$ will be given by the data of $\mathfrak{d}=k-l$.

Lemma 1. If $t$ is a crossing value, one of the following two cases occurs:

- 1st case. There is exactly one ordered pair $(m, \mathfrak{s}), 1 \leq \mathfrak{s} \leq 2 N-3$ verifying (24); we denote it $(m(t), \mathfrak{s}(t))$ and $\mathfrak{s}(t)$ is either $N-2, N-1$ or $N$.

- 2nd case. There exist exactly two $(m, \mathfrak{s})$ 's satisfying (24) with $1 \leq \mathfrak{s} \leq$ $2 N-3 ;$ we denote them $(m(t), \mathfrak{s}(t))$ and $(m(t)+q, \mathfrak{s}(t)+N)$.

Proof. We let $(m(t), \mathfrak{s}(t))$ be the ordered pair such that $\mathfrak{s}(t)$ is the smallest $\mathfrak{s}$ for the $(m, \mathfrak{s})$ 's verifying $(24)$ and $(25)$. If $\left(m_{1}, \mathfrak{s}_{1}\right)$ and $\left(m_{2}, \mathfrak{s}_{2}\right)$ both verify (24) for the same $t$, we have

$$
q\left(\mathfrak{s}_{1}-\mathfrak{s}_{2}\right)=N\left(m_{2}-m_{1}\right)
$$


Since $q$ and $N$ are mutually prime, it follows that, for some integer $a$,

$$
\mathfrak{s}_{2}=\mathfrak{s}_{1}+a N \quad m_{2}=m_{1}+a q
$$

Since $\mathfrak{s}(t)$ is the smallest one, it verifies

$$
\mathfrak{s}(t) \leq N .
$$

If we are in the 1st case of the Lemma 1, i.e. a single $(m, \mathfrak{s})$, we derive from (27) that $\mathfrak{s}(t)+N$ does not verify (25), i.e.

$$
\mathfrak{s}(t)+N>2 N-3
$$

Putting together (28) and (29), we get

$$
N-2 \leq \mathfrak{s}(t) \leq N
$$

which concludes the proof of the 1st case.

The 2nd case is clear: since $\mathfrak{s}(t)+2 N>2 N-3, \mathfrak{s}(t)+N$ is the only other integer $\mathfrak{s}$ in $[1,2 N-3]$ which can appear in (24); the corresponding $m$ is $m(t)+q$.

Lemma 1 told us which $\mathfrak{s}$ 's and $m$ occur for crossing points $(t, y)$ above a crossing value $t$ : we now find the $k, l$ 's such that $\mathfrak{s}=k+l$ and derive the $\sigma_{i}^{ \pm}$'s corresponding to the $(t, y)$ 's.

Definition 2. Let $P=(t, y)$ be a crossing point; we denote by $i(P) \in$ $\{1, \ldots, N-1\}$ the corresponding generator subscript, i.e. $P$ is represented by $\sigma_{i(P)}$ or $\sigma_{i(P)}^{-1}$.

Lemma 2. Let $t$ be a crossing value of $B_{N, q, p}$.

1. The point $P=(t, y)$ is a crossing point of the braid shadow if and only if

$$
y=(-1)^{m(t)} \cos \left(\frac{q \mathfrak{d}}{N} \pi\right)
$$

where $\mathfrak{d}$ is any integer in $[1, \ldots, N-1]$ of the same parity as $\mathfrak{s}(t)$

2. To determine $i(P)$, we do the Euclidean division of qd by $2 N$

$$
q \mathfrak{d}=2 N n+w
$$

with $n \geq 0,-N<w<N$. 
(a) If $m(t)$ is even,

$$
i(P)=i\left(t, \cos \left(\frac{q \mathfrak{d}}{N} \pi\right)\right)=|w|
$$

(b) If $m(t)$ is odd,

$$
i(P)=i\left(t,-\cos \left(\frac{q \mathfrak{d}}{N} \pi\right)\right)=N-|w|
$$

Proof. Proof of 1: we treat separately the two cases of Lemma 1.

1. 1st case: a single ordered pair $(m, \mathfrak{s})$.

Let $k, l$ such that $k+l=\mathfrak{s}(t)$ and assume that $l<k$; note that $\mathfrak{d}=k-l$ has the parity of $\mathfrak{s}(t)$.

(a) If $\mathfrak{s}(t)=N-1$, the smallest possible value for $l$ is 0 and $k-l$ runs through all integers $\mathfrak{d}, 1 \leq \mathfrak{d} \leq N-1$ with the parity of $\mathfrak{s}(t)=N-1$.

(b) If $\mathfrak{s}(t)=N-2($ resp. $\mathfrak{s}(t)=N)$, the smallest value for $l$ is 0 (resp. 1 ) and $\mathfrak{d}$ runs through the integers in $[1, N-2]$ with the parity of $N-2$ or $N$; since $N-1$ has parity opposite to $N$ and $N-2$, we can actually assume $\mathfrak{d}$ in $[1, N-1]$.

To derive (30), we plug (24) into

$$
y=\sin \left(\frac{2 \pi}{N} q(t+k)\right)
$$

2. 2nd case: two ordered pairs: $(m(t), \mathfrak{s}(t))$ and $(m(t)+q, \mathfrak{s}(t)+N)$.

(a) We first consider the $k$, l's such that $l<k$ and $k+l=\mathfrak{s}(t)$. As above, $k-l$ runs through the integers $\mathfrak{d}$ with the parity of $\mathfrak{s}(t)$ and such that

$$
1 \leq \mathfrak{d} \leq \mathfrak{s}(t)
$$

(b) If $k+l=\mathfrak{s}(t)+N$, we look at $\mathfrak{d}=k-l$ 's with $l<k$ :

$$
l=\mathfrak{s}(t)+N-k \geq \mathfrak{s}(t)+N-(N-1)=\mathfrak{s}(t)+1
$$

hence $\mathfrak{d}=\mathfrak{s}(t)+N-2 l \leq \mathfrak{s}(t)+N-2 \mathfrak{s}(t)-2=N-\mathfrak{s}(t)-2$ and 


$$
1 \leq \mathfrak{d} \leq N-\mathfrak{s}(t)-2
$$

Moreover every integer in $\mathfrak{d} \in[1, N-\mathfrak{s}(t)-2]$ with the parity of $\mathfrak{s}(t)+N$ is a legitimate $\mathfrak{d}$, i.e. there exist $k, l$ in $\{1, \ldots, N-1\}$ with $\mathfrak{d}=k-l$ and $k+l=\mathfrak{s}(t)+N$; for example $N-\mathfrak{s}(t)-2=$ $(N-1)-(\mathfrak{s}(t)+1)$.

Using (34), we derive the $y$-coordinate of the crossing point:

$$
y=(-1)^{m(t)}(-1)^{q} \cos \left(\pi \frac{q \mathfrak{d}}{N}\right)=(-1)^{m(t)} \cos \left(\pi \frac{q \tilde{\mathfrak{d}}}{N}\right)
$$

where $\tilde{\mathfrak{d}}=N-\mathfrak{d} ;$ if $\mathfrak{d}$ verifies (36), then

$$
\mathfrak{s}(t)+2 \leq \tilde{\mathfrak{d}} \leq N-1
$$

Since $\mathfrak{d}$ has the parity of $\mathfrak{s}(t)+N, \tilde{\mathfrak{d}}$ has the parity of $\mathfrak{s}(t)$.

Putting together the intervals (35) and (38) concludes the proof of the 2nd case.

Proof of 2. We derive from (31) that

$$
\cos \left(\pi \frac{q \mathfrak{d}}{N}\right)=\cos \left(\pi \frac{w}{N}\right)=\cos \left(\pi \frac{|w|}{N}\right)
$$

so 2 (a) of Lemma 2 follows from the fact that the function cos is decreasing on $(0, \pi)$ :

$$
\cos \frac{\pi}{N}>\cos \frac{2 \pi}{N}>\ldots>\cos \frac{(N-1) \pi}{N} .
$$

and 2 (b) of Lemma 2 follows from

$$
-\cos \left(\pi \frac{q \mathfrak{d}}{N}\right)=-\cos \left(\pi \frac{w}{N}\right)=-\cos \left(\pi \frac{|w|}{N}\right)=\cos \left(\pi \frac{N-|w|}{N}\right) .
$$

To see how many crossing points $(t, y)$ occur above $t$, i.e. how many values (30) takes for a given $t$, we notice the following.

- If $u, v \in\{1, \ldots, N-1\}$ and $\cos \left(\pi \frac{q u}{N}\right)=\cos \left(\pi \frac{q v}{N}\right)$, then $u=v$.

- If $u, v \in\{1, \ldots, N-1\}$ and $\cos \left(\pi \frac{q u}{N}\right)=-\cos \left(\pi \frac{q v}{N}\right)$, then $u=N-v$. 
So the sets $\left\{\cos \left(\pi \frac{q u}{N}\right)\right.$ with $\left.1 \leq u \leq N-1\right\}$ and $\left\{-\cos \left(\pi \frac{q u}{N}\right)\right.$ with $1 \leq u \leq$ $N-1\}$ are identical. We derive from Lemma 2

Corollary 2. Let $t$ be a crossing value. The indices $i(P)$ 's for the crossing points $P$ 's above $t$ are all the $i$ 's in $\{1, \ldots, N-1\}$ and with

1. the parity of $\mathfrak{d}$ and $\mathfrak{s}(t)$ if $m(t)$ is even

2. the parity of $\mathfrak{d}+N$ and $\mathfrak{s}(t)+N$ if $m(t)$ is odd.

\subsubsection{The sign of the crossing points}

We now compute the sign of the crossing points described in Lemma 2.

Lemma 3. The sign of the crossing point corresponding to the $(t, y)$ appearing in (30) is given by

$$
\Sigma(t, y)=(-1)^{m(t)} \sigma\left(p \frac{m(t)}{q}+\frac{p}{2 q}+\frac{2 p \phi}{N}\right) \sigma\left(\frac{q \mathfrak{d}}{N}\right) \sigma\left(\frac{p \mathfrak{d}}{N}\right)
$$

where $\sigma(r)$ is the parity of the integer part:

$$
\sigma(r)=(-1)^{[r]}
$$

Proof. We recall (Lemma 1) that $k+l=\mathfrak{s}(t)$ or $k+l=\mathfrak{s}(t)+N$.

If $k+l=\mathfrak{s}(t)$ and $\mathfrak{d}=k-l$, then (39) is just the formula (23) for the sign of a crossing point. So we assume that $k+l=\mathfrak{s}(t)+N$ : we have seen above that $y$ is given by (37) with $k-l=N-\tilde{\mathfrak{d}}$ and we write (23) for the sign of the crossing point

$$
\begin{gathered}
(-1)^{m(t)+q} \sigma\left(q\left(\frac{N-\tilde{\mathfrak{d}}}{N}\right)\right) \sigma\left(p\left(\frac{N-\tilde{\mathfrak{d}}}{N}\right)\right) \sigma\left(p \frac{m(t)+q}{q}+\frac{p}{2 q}+\frac{2 p \phi}{N}\right) \\
=(-1)^{m(t)} \sigma\left(q \frac{\tilde{\mathfrak{d}}}{N}\right) \sigma\left(p \frac{\tilde{\mathfrak{d}}}{N}\right) \sigma\left(p \frac{m(t)}{q}+\frac{p}{2 q}+\frac{2 p \phi}{N}\right)
\end{gathered}
$$

We write the last two factors of $(39)$ in terms of $i(P)$ : 
Lemma 4. We let $P=\left(t,(-1)^{m(t)} \cos \pi \frac{q \mathfrak{d}}{N}\right)$ be a crossing point of $B_{N, q, p}$ with $m(t), \mathfrak{d}$ and $w$ as in 2) of Lemma 2. Then

$$
\sigma\left(\frac{q \mathfrak{d}}{N}\right) \sigma\left(\frac{p \mathfrak{d}}{N}\right)=\left\{\begin{array}{c}
\sigma\left(\frac{B p i(P)}{N}\right) \text { if } p \text { is odd } \\
(-1)^{m(t)} \sigma\left(\frac{B p i(P)}{N}\right) \text { if } p \text { is even }
\end{array}\right.
$$

Proof. It follows from (31) that

$$
\sigma\left(\frac{q \mathfrak{d}}{N}\right)=\sigma\left(\frac{w}{N}\right)
$$

We recall (13), namely $2 N A+B q=1$, hence $\mathfrak{d}=2 N A \mathfrak{d}+B q \mathfrak{d}$; putting this together with the Euclidean division in (31), we have

$$
\mathfrak{d}=2 N A \mathfrak{d}+2 N n B+B w
$$

Thus

$$
\sigma\left(\frac{p \mathfrak{d}}{N}\right)=\sigma\left(\frac{p B w}{N}\right)
$$

Now

$$
\sigma\left(\frac{w}{N}\right) \sigma\left(\frac{p B w}{N}\right)=\sigma\left(\frac{|w|}{N}\right) \sigma\left(\frac{p B|w|}{N}\right)=\sigma\left(\frac{p B|w|}{N}\right)
$$

- If $m(t)$ is even, then $i(P)=|w|$ and the Lemma is proved.

- If $m(t)$ is odd, we use the fact that $B$ is odd to write

$$
\sigma\left(\frac{p B|w|}{N}\right)=\sigma\left(\frac{p B(N-i(P))|w|}{N}\right)=\left\{\begin{array}{cl}
\sigma\left(\frac{p B i(P)}{N}\right) & \text { if } p \text { is odd } \\
-\sigma\left(\frac{p B i(P)}{N}\right) & \text { if } p \text { is even }
\end{array}\right.
$$

It follows from Lemma 4 that the sign $\Sigma(P)$ given in (39) of a crossing point $P$ of crossing value $t$ is ( $\epsilon$ has been defined in (14) above)

- $\sigma\left(p \frac{m(t)}{q}+\frac{p}{2 q}+\frac{2 p \phi}{N}\right) \epsilon(N, q, p)(i)$ if $p$ is even

- $\sigma\left(p \frac{m(t)}{q}+\frac{p}{2 q}+\frac{2 p \phi}{N}\right)(-1)^{m(t)} \epsilon(N, q, p)(i)$ if $p$ is odd.

We recall (see the formulae (15) in the Main Theorem) that the \pm 1 -exponent of a $\sigma_{i}$ in $\alpha_{N, q, p}$ or $\beta_{N, q, p}$ is $\epsilon(N, q, p)(i)$, hence 
Lemma 5. The \pm 1 -exponent of the $\alpha_{N, q, p}$ or $\beta_{N, q, p}$ corresponding to a crossing value $t$ in $B_{N, q, p}$ is

1. $\sigma\left(p \frac{m(t)}{q}+\frac{p}{2 q}+\frac{2 p \phi}{N}\right)$ if $p$ is even

2. $(-1)^{m(k)} \sigma\left(p \frac{m(t)}{q}+\frac{p}{2 q}+\frac{2 p \phi}{N}\right)$ if $p$ is odd.

The formulae in Lemma 5 depend on $m(t)$, where $t$ goes through the $2 q$ crossing values. If $t$ is the $h$-th crossing value, for $h=1, \ldots, 2 q$, we want to have $m(t)$ directly as an expression in $h$ so we number the crossing values

$$
t_{1}<t_{2}<\ldots<t_{q}<\ldots<t_{2 q}
$$

and for any integer $k$, with $1 \leq k \leq 2 q$, we let

$$
m(k)=m\left(t_{k}\right) \quad \mathfrak{s}(k)=\mathfrak{s}\left(t_{k}\right)
$$

If $t_{k}$ and $t_{k+1}$ are two consecutive crossing values, we derive from (24)

$$
t_{k+1}-t_{k}=\frac{1}{2 q}[q(\mathfrak{s}(k)-\mathfrak{s}(k+1))+N(m(k+1)-m(k))] \geq \frac{1}{2 q}
$$

Since there are $2 q$ crossing values in $[\eta, 1+\eta],(48)$ is an equality and we have

$$
q(\mathfrak{s}(k)-\mathfrak{s}(k+1))+N(m(k+1)-m(k))=1
$$

We note in passing that, if we plug (49) into Proposition 2, we get the confirmation of the obvious fact

Lemma 6 . The crossing points above $t_{k}$ and $t_{k+1}$ are represented by $\sigma_{i}^{ \pm 1}$ 's with $i$ 's of opposite parities.

We now confront (49) with $2 N A+B q=1$ and derive the existence of an integer $\nu_{k}$ such that

$$
m(k+1)-m(k)=\nu_{k} q+2 A .
$$

Thus, for any $k$, there exists an integer $a_{k}$ such that

$$
m(k)=m(1)+a_{k} q+2(k-1) A
$$




$$
(-1)^{m(k)}=(-1)^{m(1)}(-1)^{a_{k}}
$$

Define $\phi_{0}$ by

$$
p \frac{m(1)}{q}-\frac{2 A p}{q}+\frac{p}{2 q}+\frac{2 p \phi_{0}}{N}=0
$$

We see (23) that $\phi_{0}$ is a critical phase, i.e. a phase for which the knot $K(N, p, q, \phi)$ is singular. So we pick a phase

$$
\phi=\phi_{0}+\xi
$$

where $\xi$ is a very small positive number. Using (50), we rewrite

$$
\sigma\left(p \frac{m(t)}{q}+\frac{p}{2 q}+\frac{2 p \phi}{N}\right)=(-1)^{a_{k} p}(-1)^{\left[\frac{2 A p}{q} k+\xi\right]}
$$

It follows from Lemma 5 and equations (50), (54) that the exponent of the $\alpha_{N, q, p}$ or $\beta_{N, q, p}$ at the $k$-th crossing value is

$$
\left\{\begin{array}{c}
(-1)^{m(1)}(-1)^{\left[\frac{2 A p}{q} k+\xi\right]} \text { if } p \text { is odd } \\
(-1)^{\left[\frac{2 A p}{q} k+\xi\right]} \text { if } p \text { is even }
\end{array}\right.
$$

Since we are working up to mirror transformation, we assume

$$
(-1)^{m(1)}=1 \text {. }
$$

We now conclude: the expression $\left[\frac{2 A p}{q} k+\xi\right]$ is equal to 1 for $k=q, 2 q$ and equal to $\lambda(k)$ for the other $k$ 's. Going back to the statement of the Main Theorem, this gives us the exponent for the $k$-th crossing values with $k \leq q$ or $k=2 q$. We settle the case of the $k$ 's with $q<k<2 q$ by noticing that

$$
\lambda(2 q-k)=-\lambda(k) .
$$

\section{The four-genus}

\section{$5.1 \quad$ Ribbon knots}

A ribbon knot in $\mathbb{S}^{3}$ bounds a disk in $\mathbb{S}^{3}$ with only ribbon singularities; equivalently it bounds an embedded disk in $\mathbb{B}^{4}$ with does not have local maxima for the distance to the origin of $\mathbb{B}^{4}$. Thus a ribbon knot is slice, i.e. its 4genus is zero; the long-standing Slice-Ribbon conjecture asks if the converse is true. 


\subsubsection{Proof of Theorem 1}

Th. 1 has been proved by Lamm and also follows from his more general construction of ribbon symmetric unions ([La 2], $[\mathrm{K}-\mathrm{T}]$ ). His proof is fairly allusive so we felt it would be useful to give a more detailed proof.

We recall the well-known fact:

Proposition 5. Let $K$ be a knot in $\mathbb{R}^{3}$ which is symmetric with respect to a plane $P$ in $\mathbb{R}^{3}$; then it is ribbon.

Proof. We endow $\mathbb{R}^{3}$ with the frame $O x y z$ and assume that $P$ is defined by the equation $x=0$. By genericity arguments, we assume

1. $K$ meets $P$ at a finite number of points

2. outside of $P, K$ is never tangent to the direction of $O x$.

Since $K$ has one component, it meets $P$ at exactly two points.

We let $K_{+}$(resp. $K_{-}$) be the intersection of $K$ with the half-space of $\mathbb{R}^{3}$ defined by $z \geq 0$ (resp. $z \leq 0$ ): $K_{+}$and $K_{-}$are both diffeomorphic to a closed interval.

Letting $S$ be the symmetry in $\mathbb{R}^{3}$ with respect to $P$, we let

$$
\begin{gathered}
\Phi=[0,1] \times K_{+} \longrightarrow \mathbb{R}^{3} \\
(t, X) \mapsto t X+(1-t) S(X)
\end{gathered}
$$

The self-intersections of $\Phi$ are given by the data of $t_{1}, t_{2}, X_{1}, X_{2}$ such that

$$
t_{1} X_{1}+\left(1-t_{1}\right) S\left(X_{1}\right)=t_{2} X_{2}+\left(1-t_{2}\right) S\left(X_{2}\right)
$$

We denote by $\left(x_{i}, y_{i}, z_{i}\right), i=1,2$ the coordinates of $X_{i}$. Since $S\left(x_{i}, y_{i}, z_{i}\right)=$ $\left(-x_{i}, y_{i}, z_{i}\right),(56)$ implies that

$$
y_{1}=y_{2}, z_{1}=z_{2} .
$$

Thus the line segments $I_{1}=X_{1} S\left(X_{1}\right)$ and $I_{2}=X_{2} S\left(X_{2}\right)$ are both included in the line which is defined by the equations $y=y_{1}, z=z_{1}$. Moreover, one of them is included in the other one and we have a ribbon singularity. 

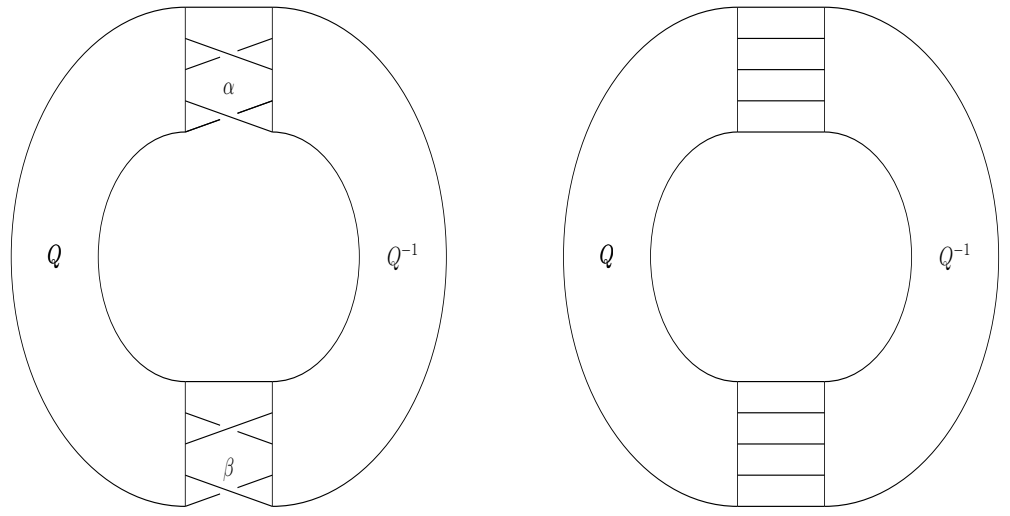

Figure 8: $B(5, q, p)$ and the link $L$

The Main Theorem tells us that, if $p$ and $q$ are mutually prime, with $q$ odd, $B_{N, q, p}$ is as in Fig. 8.

If $N$ is the number of strands, there are $N-1$ half-twist tangles connecting $Q$ and $Q^{-1}$; we replace them by $N-1$ trivial tangles and get the $N$-component link $L$ of Fig. 8 which is symmetric w.r.t. a plane.

Proposition 5 tells us that $L$ bounds $N$ ribbon disks $D_{1}, \ldots, D_{N}$; and the same arguments show us that two of these disks only have ribbon-type intersection.

We now connect each $D_{i}$ to $D_{i+1}$ by a half-twisted band bounded by the half-twist tangle of Fig. 8. The resulting surface is a topological disk with only ribbon singularities.

\subsection{General case: proof of Theorem 2}

We use an idea by Brandenbursky and Kedra ([B-K]). If $b$ is a $N$-braid, we denote by $\hat{b}$ the link obtained by closing the braid $b$. If $b_{1}$ and $b_{2}$ are $N$-two braids, $[\mathrm{B}-\mathrm{K}]$ constructed a cobordism of Euler characteristic $-N$ between the closure of the product $\widehat{b_{1} b_{2}}$ and the disjoint union of the closures $\widehat{b_{1}} \sqcup \widehat{b_{2}}$. Letting $\tilde{q}=\frac{q}{N}, \tilde{p}=\frac{p}{N}$, we recall that

$$
B_{N, q, p}=B_{N, \tilde{q}, \tilde{p}}^{d}
$$

Applying [B-K]'s result $d$ times, we derive a cobordism in $\mathbb{B}^{4}$ of Euler charac-

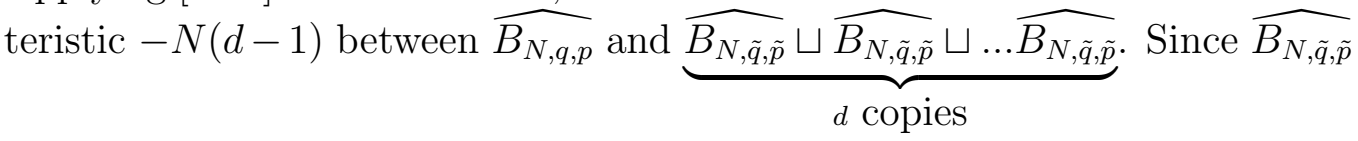


is a ribbon knot (Theorem 1 ), it bounds an embedded disk in $\mathbb{B}^{4}$. Thus $\widehat{B_{N, q, p}}$ bounds a surface of Euler characteristic

$$
-d(N-1)+d=1-(d-1)(N-1) .
$$

We recover the formula (9) for the genus and Theorem 2 is proved.

\subsection{Quasipositive knots: proof of Proposition 2}

Lee Rudolph (see [Ru] for details) defines a braid $\gamma \in \mathbf{B}_{N}$ to be quasipositive if it is a product of conjugates $w \sigma_{i} w^{-1}$ of positive braid generators, i.e.

$$
\gamma=w_{1} \sigma_{i_{1}} w_{1}^{-1} w_{2} \sigma_{i_{2}} w_{2}^{-1} \ldots w_{k} \sigma_{i_{k}} w_{k}^{-1}
$$

Theorem 3. ([Ru]) If $\gamma$ is a quasipositive braid written as in (57) closing in a knot $\hat{\gamma}$, its four-genus verifies

$$
1-2 g_{4}(\hat{\gamma})=N-k
$$

It is easy to check that under the assumptions of Proposition 2, the exponents of all the $\sigma_{2 k}$ 's and $\sigma_{2 k+1}$ 's appearing respectively in $\alpha_{N, q, p}$ and $\beta_{N, q, p}$ are all of the same sign. Since we are working up to mirror symmetry, we can assume all these exponents to be equal to 1 ; thus

$$
\alpha_{N, q, p}=\prod_{2 \leq 2 k \leq N-1} \sigma_{2 k} \quad \beta_{N, q, p}=\prod_{1 \leq 2 k+1 \leq N-1} \sigma_{2 k+1}
$$

Hence $B_{N, p, q}=\left(Q \alpha_{N, q, p} Q^{-1} \beta_{N, q, p}\right)^{d}$ is a quasipositive braid; Theorem 3 tells us that $1-2 g_{4}(K(N, q, p))=N-d(N-1)$ and Proposition 2 follows.

\section{Trivial knots: proof of Proposition 4}

\subsection{The knot $K(N, q, q+N)$ is trivial}

We set

$$
A=\prod_{1 \leq 2 k \leq N} \sigma_{2 k} \quad B=\prod_{1 \leq 2 k+1 \leq N} \sigma_{2 k+1}
$$

Lemma 7.

$$
B_{N, q, q+N}=A(B A)^{\frac{q-1}{2}}\left(B^{-1} A^{-1}\right)^{\frac{q-1}{2}} B^{-1}
$$


The main thing to note about this formula is that all the positive generators are on one side and all the negative generators are on the other side.

Proof. We use the Main Theorem. It assumes that $q$ is odd but in the present case, if $q$ is even, $N$ has to be odd, hence $q+N$ is odd and we switch $q$ and $q+N$ to apply the theorem. We compute

$$
\epsilon(i)=(-1)^{i} \quad \lambda(k)=(-1)^{k}
$$

Thus $\alpha_{N, q, q+N}=\prod_{1 \leq 2 k \leq N} \sigma_{2 k}=A$ and $\beta_{N, q, q+N}=\prod_{1 \leq 2 k+1 \leq N} \sigma_{2 k+1}^{-1}=B^{-1}$. We conclude by noticing that

$$
(A B)^{\frac{q-1}{2}} A=A(B A)^{\frac{q-1}{2}}
$$

We construct a trivial pure braid $\mathcal{B}_{N}$; we will show that $B_{N, N+q, q}$ is the product of a power of $\mathcal{B}_{N}$ and of a piece of $\mathcal{B}_{N}$.

If $N=2 k$ is even, we let

$$
\mathcal{B}_{N}=(B A)^{k}\left(B^{-1} A^{-1}\right)^{k} .
$$

If $N=2 k+1$ is odd, we let

$$
\mathcal{B}_{N}=(B A)^{k} B A^{-1}\left(B^{-1} A^{-1}\right)^{k} .
$$

In both cases, we check that the corresponding permutation between the endpoints of the braid is the identity, thus $\mathcal{B}_{N}$ is a pure braid.

To prove that it is a trivial braid, we discuss when one strand of $\mathcal{B}_{N}$ is above another one; so let us fix some terminology.

We number the strands of $\mathcal{B}_{N}$ : the $j$-th strand, $0 \leq j \leq N-1$ is the strand starting at the $(j+1)$-th point on the left (the points being counted from top to bottom).

We say that the $j$-th strand is above the $k$-th strand if, wherever there is a crossing point between these two strands, the $j$-th strand is above the $k$ th strand. As an example, in Fig. 9, the red strand is above all the other strands. 


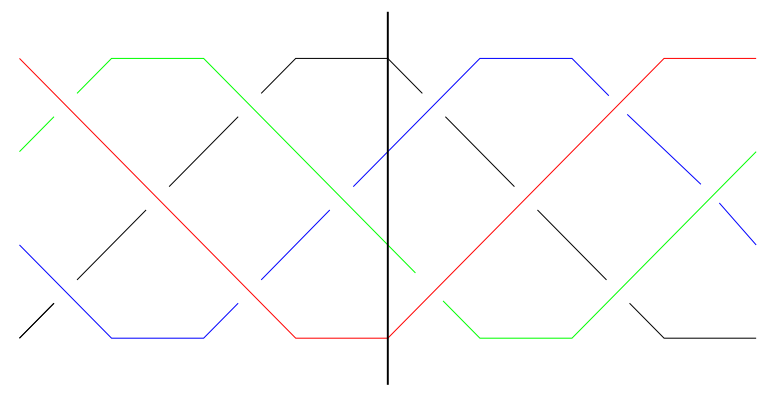

Figure 9: $B_{4,4}=\sigma_{1} \sigma_{3} \sigma_{2} \sigma_{1} \sigma_{3} \sigma_{2} \sigma_{1}^{-1} \sigma_{3}^{-1} \sigma_{2}^{-1} \sigma_{1}^{-1} \sigma_{3}^{-1} \sigma_{2}^{-1}$

Lemma 8. If $j, k$ are two integers with $0 \leq j<k \leq N-1$, the $j$-th strand of $\mathcal{B}_{N}$ is above the $k$-th strand. Thus $\mathcal{B}_{N}$ closes in $N$ unlinked trivial links, i.e. $\mathcal{B}_{N}=1$.

The figure 9 illustrates the lemma.

Proof. We describe the strands of $\mathcal{B}_{N}$ in the braid shadow, i.e. their projection to the $x y$. We endow the plane with a coordinate $O x y$ such that the $j$-th strand starts at $(0,-j)$ and ends at $(2 N,-j)$. The upper left point has coordinates $(0,0)$ (in Figure 9 it is the starting point of the red strand). We say that a strand is ascending, denoted $\nearrow$ (resp. descending, denoted $\searrow$ ) if it has a +1 (resp. -1 ) slope. It is horizontal, denoted $\longrightarrow$, when the slope is 0 .

We describe here the $k$-strands for $k$ odd (the case of an even $k$ is similar): it goes up and down as follows

1. \} \text { from } ( 0 , - k ) \text { to } ( N - 1 - k , - ( N - 1 ) )

2. $\longrightarrow$ from $(N-1-k,-(N-1))$ to $(N-k,-(N-1))$

3. $\nearrow$ from $(N-k,-(N-1))$ to $(2 N-1-k, 0)$

4. $\longrightarrow$ from $(2 N-1-k, 0)$ to $(2 N-k, 0)$

5. \from $(2 N-k, 0)$ to $(2 N,-k)$

Assume now that the $k$-th strand is above the $j$-th strand at a crossing point $(x, y)$. Assuming that $j$ is odd (the even case is similar), one of the following two cases occurs 
1. $0 \leq x \leq N$ and the $k$-th (resp. $j$-th) strand is $\searrow$ (resp. $\nearrow$ ). Then the $k$-th strand is as 1 . above and the $j$-th strand is as 3 .

2. $N \leq x \leq 2 N$ and the $k$-th (resp. $j$-th) strand $\nearrow$ (resp. \). Then the $k$-th strand is as 3 . above and the $j$-th strand is as 5 .

In both cases it is easy to check that $k<j$.

We conclude the proof of the proposition in the case when $N$ is even; the odd case is similar. We derive from Lemma 8 that for an $n>k$,

$$
\begin{gathered}
A(B A)^{n}\left(B^{-1} A^{-1}\right)^{n} B=A(B A)^{n-k}(B A)^{k}\left(B^{-1} A^{-1}\right)^{k}\left(B^{-1} A^{-1}\right)^{n-k} B \\
=A(B A)^{n-k}\left(B^{-1} A^{-1}\right)^{n-k} B .
\end{gathered}
$$

Thus, if $b$ is the remainder of the division of $\frac{q-1}{2}$ by $k$, we have

$$
B_{N, q, q+N}=A(B A)^{b}\left(B^{-1} A^{-1}\right)^{b} B
$$

The braid (64) is a piece of the braid $(B A)^{k}\left(B^{-1} A^{-1}\right)^{k}$ where the $i$-th strand is above the $j$-th strands, for $j>i$. Thus the same is true for (64) which closes therefore in a trivial knot.

\subsection{The knot $K(N, 1, p)$ is trivial}

This follows from the Main Theorem. We can also prove it directly by computing the crossing points and their sign: we see that every $\sigma_{i}^{ \pm}$appears once and only once in the braid $B_{N, 1, p}$ and so braid represents a trivial knot.

\subsection{The other knots of Proposition 4}

We have now seen two cases where $K(N, q, p)$ is trivial. We know that $K(N, q, p)$ and $K(N, p, q)$ are isotopic; and $K(N, q, k)$ and $K(N, q, 2 q N+$ $k)$ (resp. $K(N, q, 2 q N-k)$ ) are isotopic (resp. mirror image of one another). Thus we can get more examples of trivial knots, e.g. $K(3,5,29)$ and $K(3,5,31)$. 


\section{Lists of Jones polynomials}

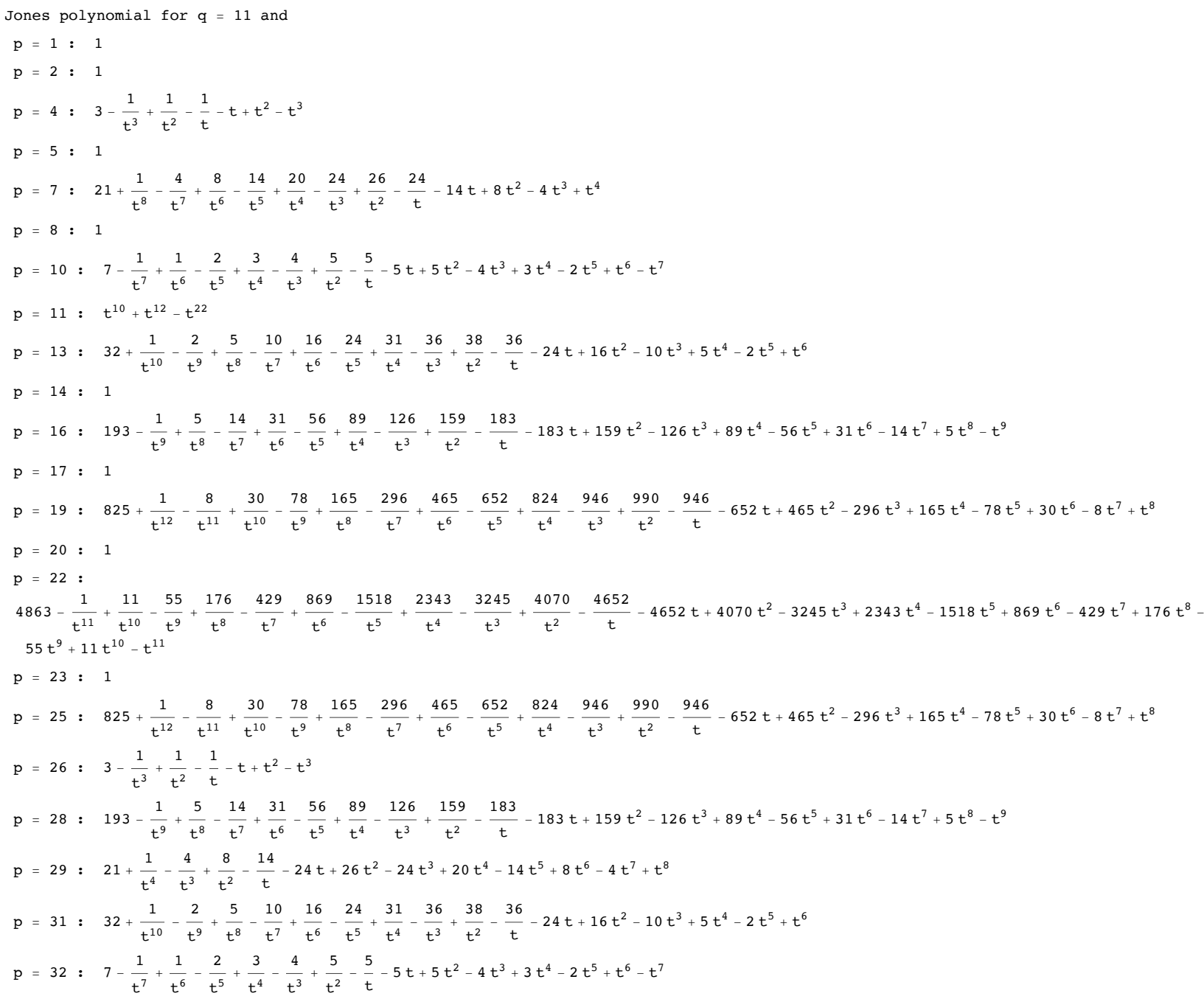

Figure 10: List of Jones polynomials of knots $K(3,11, p)$ 


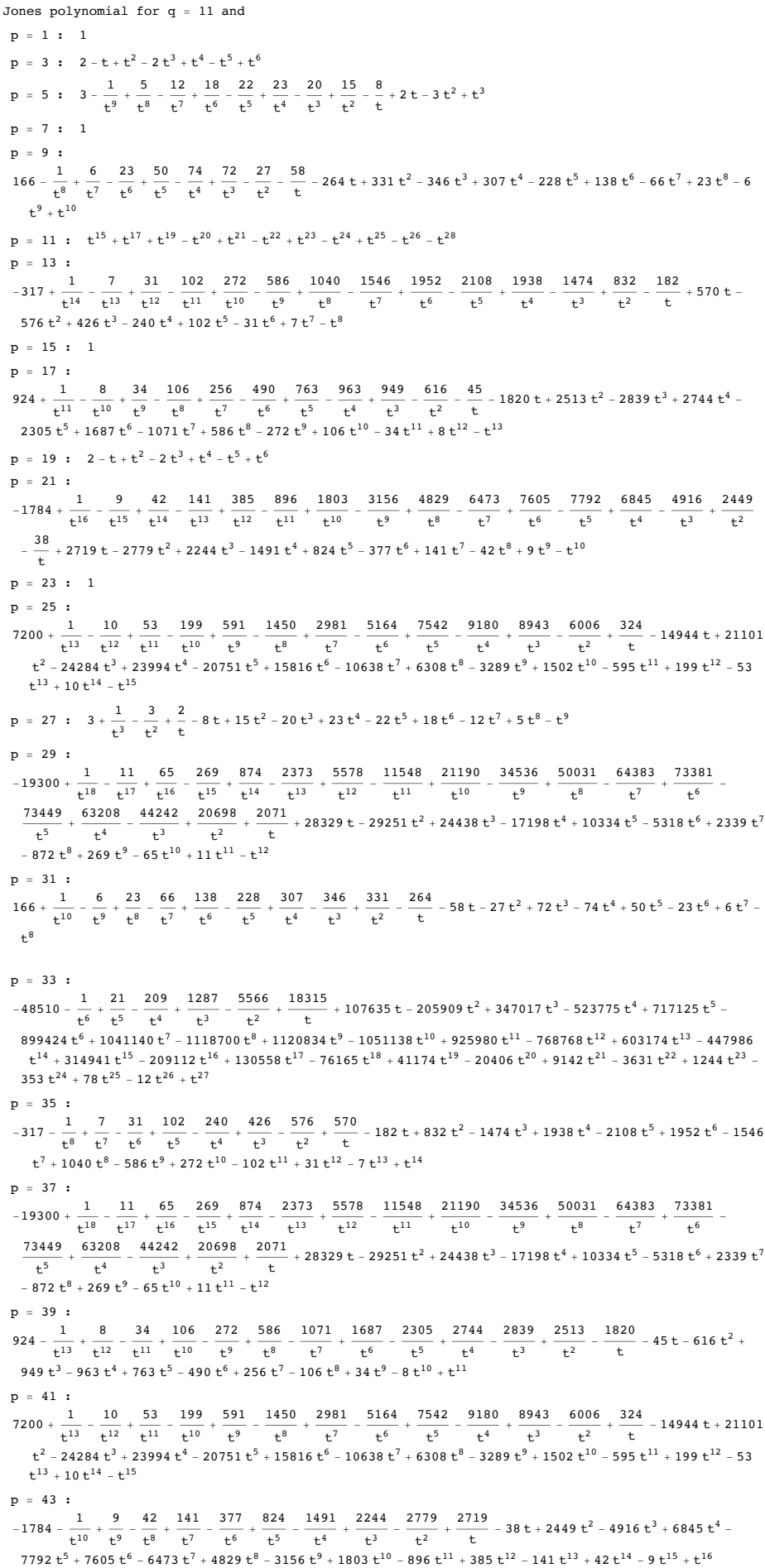

Figure 11: List of Jones polynomials of knots $K(4,11, p)$ 


\section{Appendix}

We give a better proof of the following fact from $[\mathrm{S}-\mathrm{V}]$ :

Proposition 6. Let $\phi_{1}$ and $\phi_{2}$ two real numbers. The knots $K\left(N, q, p, \phi_{1}\right)$ and $K\left(N, q, p, \phi_{2}\right)$ defined in (1) are either isotopic or mirror image of one another.

Proof. Without loss of generality, we assume $\phi_{1}<\phi_{2}$.

If there is no critical phase (i.e. a phase for which the knot is singular) between $\phi_{1}$ and $\phi_{2}$, the two knots are isotopic.

In $[\mathrm{S}-\mathrm{V}]$ we showed that the difference between two critical phases is of the form

$$
\frac{N}{2}\left(\frac{m}{p}+\frac{n}{q}\right)
$$

for two integers $m, n$.

Thus it is enough to prove that, for a given $\phi_{3}$, and integers $m$ and $n$, the knots $K\left(N, q, p, \phi_{3}\right)$ and $K\left(N, q, p, \phi_{3}+\frac{N}{2}\left(\frac{m}{p}+\frac{n}{q}\right)\right)$ are the same or mirror images of one another.

Consider the parametrization of $K(N, q, p, \phi)$ given in (19); we change its variable by setting

$$
s=t+\frac{N n}{2 q}
$$

and we rewrite the expression in (19)

$$
\begin{aligned}
& \left(\sin \frac{2 \pi q}{N}(t+k), \cos \frac{2 \pi p}{N}\left(t+k+\phi_{3}+\frac{N}{2}\left(\frac{m}{p}+\frac{n}{q}\right)\right)\right) \\
& =\left((-1)^{n} \sin \frac{2 \pi q}{N}(s+k),(-1)^{m} \cos \frac{2 \pi p}{N}\left(s+k+\phi_{3}\right)\right)
\end{aligned}
$$

Thus, if $m$ and $n$ have the same (resp. opposite) parities, the two knots are isotopic (resp. mirror images of one another). 


\section{References}

[br] The Liverpool knot group br9z.p, https://www.liverpool.ac.uk/ su14/knotprogs.html

[B-K] M. Brandenbursky, J. Kedra Concordance group and stable commutator length in braid groups, arXiv:1402.3191 (2014), to appear in Algebraic \& Geometric Topology (2015).

[B-Z] G. Burde, H. Zieschang, Knots, de Gruyter Studies in Math., 2nd Ed. vol. 5, Walter de Gruyter \& Co, New York, 2003.

[Co] J.H. Conway assisted by Fung, F. Y. C. The sensual (quadratic) form MAA (1997).

[Cr] P. Cromwell Knots and links Cambridge University Press, 2014.

[F-W] J. Franks, R. Williams Braids and the Jones-Conway polynomial, Trans. Amer. Math. Soc. 303 (1987) 97-108

[Ka] L.H. Kauffman On knots, PUP, 1987.

[KP] http://www.knotplot.com/

[JP] V.F.R. Jones, J.H. Przytycki Lissajous knots and billiard knots Knot theory, Banach Center Publications, 42, Inst. of Maths, Polish Acad. of Sciences, Warsaw 1998

[K-M] P. Kronheimer, T. Mrowka, The Genus of Embedded Surfaces in the Projective Plane, Math. Res. Letters 1, 797-808 (1994)

[K-T] S. Kinoshita, H. Terasaka On unions of knots, Osaka J. Math. 9 (1957), 131-153.

[L-O] C. Lamm, D. Obermeyer Billiard knots in a cylinder J. Knot Theory and its Ramifications 8(3) (1999) Vol. 353-366.

[La 1] C. Lamm Deformation of cylinder knots Fourth chapter of Ph.D. thesis Zylinder-Knoten und symmetrische Vereinigungen Bonner Mathematische Schriften 321 (1999), http://arxiv.org/pdf/1210.6639 (2012)

[La 2] C. Lamm Symmetric unions and ribbons knots, Osaka J. Math. 37 (2000), 537-550 
[M-O] X. Mo, R. Osserman, On the Gauss map and total curvature of complete minimal surfaces and an extension of Fujimoto's theorem, Jour. Diff. geom. 31 (1990), 343-355.

[M-W] M. Micallef, B. White, The structure of branch points in minimal surfaces and in pseudoholomorphic curves, Annals of Maths, 139, (1994), $35-85$

[Mi] J. Milnor, Singular points of complex hypersurfaces, Ann. of Math. Studies, PUP (1968).

[Ro] D. Rolfsen, Knots and Links, Publish or Perish, Houston, 1990.

[Ru] L. Rudolph, Quasipositivity as an obstruction to sliceness, Bull. of the AMS, 29 (1) (1993) Pages 51-59

[S-V] M. Soret, M. Ville, Singularity knots of minimal surfaces in $\mathbb{R}^{4}$, Journal of Knot theory and its ramifications, 20 (04) 2011, 513-546.

Université F. Rabelais, Dép. de Mathématiques, 37000 Tours, France, Marc.Soret@lmpt.univ-tours.fr, Marina.Ville@lmpt.univ-tours.fr 